\title{
Maximising recruitment and retention of general practices in clinical trials: a case study
}

\author{
Elizabeth Dormandy, Fred Kavalier, Jane Logan, Hilary Harris, Nola Ishmael \\ and Theresa M Marteau on behalf of the SHIFT research team
}

\author{
ABSTRACT \\ Background \\ There is limited evidence regarding the factors that \\ facilitate recruitment and retention of general practices in \\ clinical trials. It is therefore pertinent to consider the \\ factors that facilitate research in primary care. \\ Aim \\ To formulate hypotheses about effective ways of \\ recruiting and retaining practices to clinical trials, based \\ on a case study.

\section{Design of study} \\ Case study of practice recruitment and retention to a trial \\ of delivering antenatal sickle cell and thalassaemia \\ screening.

\section{Setting} \\ Two UK primary care trusts with 123 practices, with a \\ high incidence of sickle cell and thalassaemia, and high \\ levels of social deprivation.

\section{Method} \\ Practices were invited to take part in the trial using a \\ research information sheet for practices. Invitations were \\ sent to all practice managers, GPs, practice nurses, and \\ nurse practitioners. Expenses of approximately $£ 3000$ per \\ practice were available. Practices and the research team \\ signed research activity agreements, detailing a payment \\ schedule based on deliverables. Semi-structured \\ interviews were completed with 20 GPs who participated \\ in the trial. Outcome measures were the number of \\ practices recruited to, and completing, the trial. \\ Results \\ Four practices did not agree to randomisation and were \\ excluded. Of 119 eligible practices, 29 expressed an \\ interest in participation. Two practices withdrew from the \\ trial and 27 participated (two hosted pilot studies and 25 \\ completed the trial), giving a retention rate of $93 \%$ \\ (27/29). The 27 participating practices did not differ from \\ non-participating practices in list size, number of GPs, \\ social deprivation, or minority ethnic group composition \\ of the practice population.

\section{Conclusion} \\ Three factors appeared important in recruiting practices: \\ research topic, invitation method, and interest in \\ research. Three factors appeared important in retaining \\ practices: good communication, easy data-collection \\ methods, and payment upon meeting pre-agreed targets. \\ The effectiveness of these factors at facilitating \\ recruitment and retention requires assessment in \\ experimental studies. \\ Keywords \\ clinical trials; primary health care; recruitment; retention.
}

\section{INTRODUCTION}

Research activity in primary care will increase as the number of chronic conditions managed in primary care continues to rise. For example, the recent 'Roadmap' published by the Royal College of General Practitioners asserts that 'virtually all health problems could be dealt with in primary care'. ${ }^{1}$ It is therefore pertinent to consider the factors that facilitate research in primary care.

Recruiting and retaining GPs to participate in trials is challenging. ${ }^{2-4} \mathrm{~A}$ recent review identified the lack of evidence about factors associated with the recruitment to research of health professionals in primary care. ${ }^{5} \mathrm{~A}$ range of interventions, including the use of printed educational materials, financial incentives, reminders, computer prompts, and trial organisation, were described, but it was noted that there was an absence of evidence regarding their effectiveness. Experience from seven dyspepsia trials indicated that organisational strategies, such as the use of experienced researchers, methods of identifying eligible patients, GP workload, and

E Dormandy, PhD, research fellow; TM Marteau, $P h D$, CPsychol, FMed Sci, professor of health psychology, King's College London. F Kavalier, MRCP (UK), primary care geneticist/consultant, Department of Clinical Genetics, Guy's Hospital, London. J Logan, MA, MRCGP, Mawbey Group Practice, London. H Harris, FRCGP, senior partner, Brooklands Medical Practice, Manchester 1980-2007. N Ishmael, OBE, DUniv, RGH, RGN, former nursing officer, Department of Health, London, on behalf of the SHIFT research team.

Address for correspondence

Professor Theresa M Marteau, Psychology Department, 5th Floor, Thomas Guy House, Guy's Campus, King's College London, London, SE1 9RT.

E-mail: theresa.marteau@kcl.ac.uk

Submitted: 26 September 2007; Editor's response: 2 January 2008; final acceptance: 14 March 2008.

(C)British Journal of General Practice.

This article was originally online first. Cite this article as: Br J Gen Pract 2008; 58: 759-766. Advance online publication. DOI: $10.3399 /$ bjgp08X319666 


\section{How this fits in}

The focus of research into chronic conditions will shift from hospital to primary care as these conditions are increasingly managed in primary care. There is limited evidence, however, regarding the factors that facilitate recruitment and retention of general practices in clinical trials. A case study describing the recruitment and retention of 25 practices into one clinical trial is used to generate hypotheses about effective methods.

simplicity of patient eligibility criteria, may be more effective than more specific strategies at increasing recruitment in primary care..$^{5}$ In addition, concerns have been raised that the most successful recruitment strategies may lead to biased results. For example, the 'physicians recruiting physicians' strategy leads to acceptable recruitment rates but may not include representative practices. ${ }^{6}$

One example of a potentially effective approach to practice recruitment that is built on an intimate knowledge of research in primary care involves the use of research information sheets for practices. ${ }^{7}$ These provide a template for the generation of clear and succinct information about a trial or research study. Recipients report that these provide a good basis for practices to decide whether to participate in a study. ${ }^{7}$ There has, however, been no forma evaluation of their effectiveness in recruiting GPs and practices to research.

While there are several published case studies describing the recruitment of practices to trials, there is limited evidence on the strategies most likely to achieve retention of representative primary care practices to trials. ${ }^{8}$ In a US study, $22 \%$ of practices dropped out of a trial of cancer prevention in primary care within 5 months of agreeing to take part in it. ${ }^{9}$ There is a lack of evidence of factors associated with successful retention of practices in primary care trials. Failure to retain practices may also reflect a failure to recruit participants, and there is some evidence of failure to recruit participants in primary care. ${ }^{10-12}$ Prout et al interviewed nine GPs and one practice nurse after they had recruited varying numbers of children to a trial. ${ }^{13}$ Good trial organisation and simple documentation and trial procedures were reported as facilitating recruitment. Although this evidence points to features associated with successful recruitment of participants by GPs, there is limited evidence or guidance on strategies to successfully retain practices in trials.

Research networks of practices have been set up in the UK and elsewhere to facilitate the recruitment of practices to clinical trials and other research studies, and to encourage primary care workers to undertake their research. The first primary care research network in the UK was the MRC General
Practice Research Framework (MRC-GPRF), set up in 1973 for an MRC-funded trial of treatment for mild hypertension. In 1986 this was developed into a national research resource. The framework now provides research access to $10 \%$ of general practices in the UK, although these practices are not representative of UK general practices as a whole. ${ }^{14}$ The Primary Care Research Network (PCRN) was set up in March 2007 to increase the number of patients recruited or involved in research in primary care. ${ }^{15}$ Neither network, however, currently provides published guidance on recruitment and retention of primary care practices.

The aim of this article is to present a detailed case study of the methods used to recruit and retain general practices in a clinical trial conducted in primary care in order to form the basis for formulating hypotheses about effective ways of recruiting and retaining general practices to clinical trials.

\section{METHOD}

\section{Study design}

This is a descriptive case study of the recruitment and retention of general practices into a cluster randomised trial (SHIFT: Screening for Haemoglobinopathies in the First Trimester). ${ }^{16}$ The trial aimed to assess the effectiveness, feasibility, and acceptability of delivering antenatal sickle cell and thalassaemia screening in primary care. The run-in data from this trial are reported elsewhere. ${ }^{17}$

\section{Setting}

The study took place in two UK inner-city primary care trusts (PCTs) with 123 general practices. The PCT areas are ranked among the most deprived in England (6th and 13th out of 354), and about $40 \%$ of their total populations are from minority ethnic groups. ${ }^{18}$ Six per cent of pregnant women in both areas carried a clinically significant haemoglobin variant. ${ }^{19} \mathrm{~A}$ universal screening policy was in place in the PCTs at the time of the trial: antenatal sickle cell and thalassaemia screening was offered to all pregnant women regardless of ethnicity. ${ }^{20}$

\section{Measures}

Outcome measures were the number of practices participating in, and completing, the trial. The target was 24 practices, as power calculations indicated that data from 24 practices would provide sufficient power to answer the trial research question. ${ }^{16}$

\section{Sample}

The sample was all general practices in two PCTs $(n=$ 123). These practices included 123 practice managers, $450 \mathrm{GPs}, 150$ practice nurses and nurse practitioners. At least one GP from each intervention practice was 
interviewed at the end of the trial to assess GPs' experiences of participating in the trial $(n=20)$.

\section{What practices were required to do}

Practices provided anonymised data on gestational age at first visit, gestational age at testing, and demographic data (age, parity, and ethnicity) during the run-in phase of the trial. These data were collected by GPs, practice nurses, and practice managers. The run-in phase lasted for a minimum of 6 months or until data on 33 eligible pregnancies were obtained. This was followed by a minimum 7-month intervention phase, when practices offered antenatal sickle cell and thalassaemia screening to women, according to the randomisation group. The randomisation groups were:

- group 1: in primary care, when women first report their pregnancies, with partners offered testing at the same time;

- group 2: in primary care, when women first report their pregnancies, with partners offered testing later and only if women are identified as carriers;

- group 3: in community-based secondary care, when women are booked by midwives, with partners offered testing later and only if women are identified as carriers.

This was a cluster randomised trial with practice as the unit of randomisation. During the intervention phase, practices collected anonymised data as described in the run-in phase. In addition, eligible participants were asked by their GPs if the research team could contact them to invite them to take part in the trial evaluation. Women who agreed to be contacted by the research team were contacted in their preferred language, using a telephone interpreter if necessary, and consent was sought to take part in the trial evaluation. Finally, intervention practices were asked to nominate two health professionals for an interview exploring the feasibility of offering antenatal sickle cell and thalassaemia screening in primary care.

Run-in data collection started in the first practice in June 2005, and the intervention data collection was completed in the last practice in July 2007.

\section{Procedure}

Using methods described in the literature and those based on the experiences of the research team, the following strategies to recruit and retain practices in the trial were developed and implemented.

Invitation. Extensive drafting of the invitation letter and research information sheet for practices was undertaken with input from three GPs on the research team and two GPs from the participating PCTs. The information sheet was tailored to each PCT and contained information about the low proportion of women who were offered antenatal sickle cell and thalassaemia screening in the first trimester in each PCT.

Letters of invitation ( $n=723)$, together with an information sheet (Appendix 1), were sent to all practice managers, GPs, practice nurses, and nurse practitioners in the two PCTs. The invitation letters included an endorsement of the trial from the local PCT. They were tailored to each job title and signed by the trial manager, principal investigator, and a local practising GP.

The trial manager contacted every practice manager within 2 weeks of practices receiving invitation letters to assess practice interest in trial participation. All practices that expressed an interest in the trial were visited to discuss the trial in more detail.

Costs. About £3000 was available for each practice that completed the trial: the exact amount paid varied between $£ 2100$ and $£ 3900$, depending on randomisation group and practice size. This covered the administrative costs of providing anonymised data, the costs of offering antenatal sickle cell and thalassaemia screening to pregnant women, and locum costs for attending training and interviews. A payment schedule was detailed in a research activity agreement, which practices were asked to sign if they wished to participate in the trial. Monies were paid in three equal portions at three points in time: on completion of the run-in data collection, on completion of the intervention phase, and on completion of GP interviews.

Communication. Open communication between each practice and the research team was encouraged, in order to facilitate early problem solving. A link person in each practice was identified as the main practice contact for the trial. Weekly contact was maintained between this nominated person or a deputy, and a nominated person in the research team for each practice during the data-collection periods. This was the usual route of communication between the practice and research team.

All GPs, practice nurses, and nurse practitioners in each practice were invited to attend a 3-hour in-house training session for practices randomised to groups 1 or 2, and a 1-hour in-house training session for practices randomised to group 3 . The communication strategy was designed to ensure that any problems the practice had in adhering to the research protocol (identified by the practice or the research team) could be identified and solved early. 


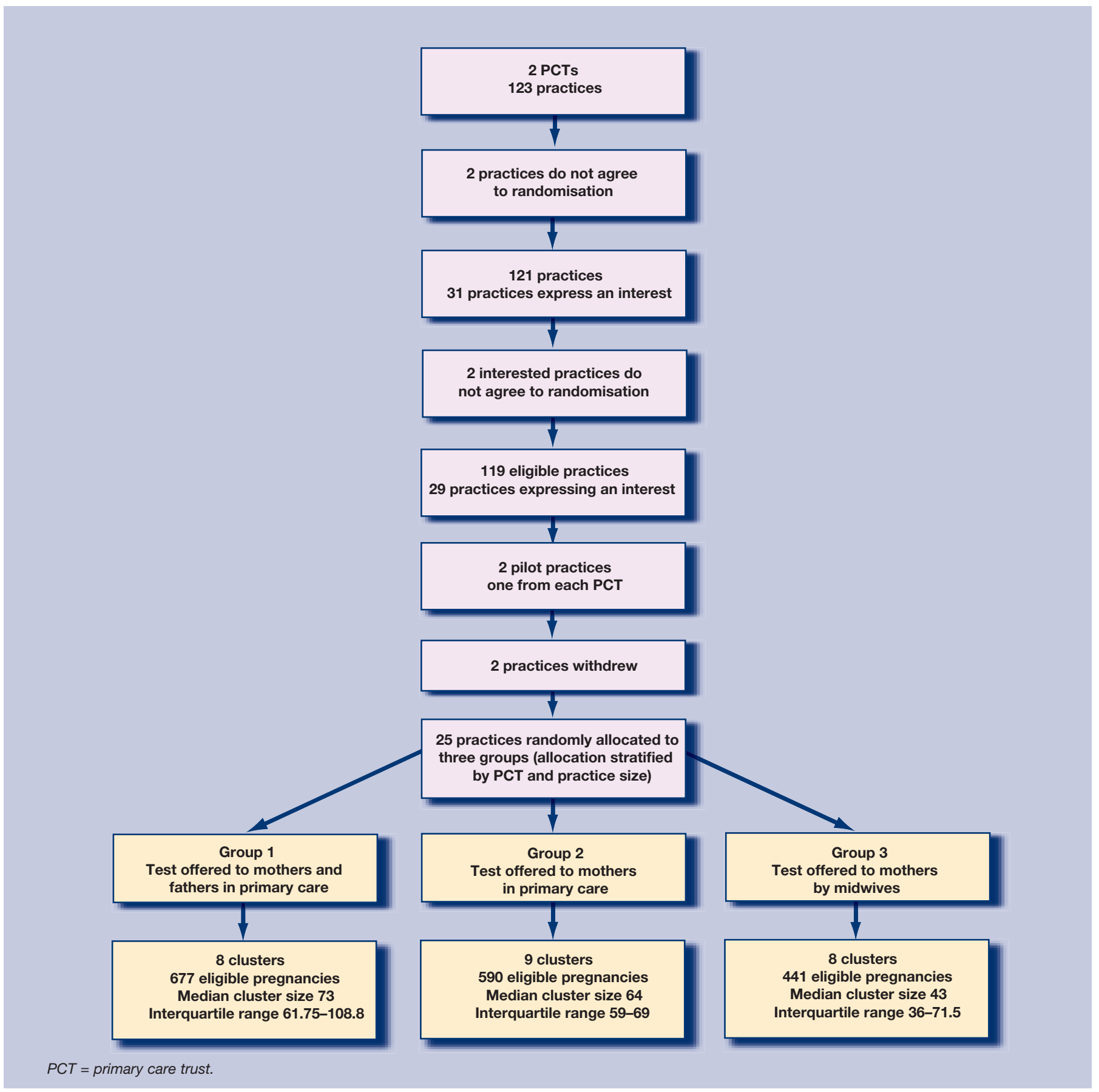

Figure 1. Flow diagram of practices in the trial.
During the 2-year trial period, three newsletters were produced to describe trial progress. These were circulated to all participating practice managers, GPs, nurse practitioners, and practice nurses. Practices were invited to contact the research team for additional training for new members of staff. Two practices requested this.

Data monitoring by the research team identified five practices that had not informed the research team of all eligible pregnancies. The trial manager visited these practices to retrain them in the research protocol. Retraining focused on reminding practices about the trial and the need to inform the research team of all eligible pregnancies.

Pilot sites. Two practices acted as pilot sites for the trial. The aim of the pilot was to identify robust methods of data collection, and assess the feasibility of trial methodology in the everyday primary care setting.

Data collection. Two methods of data collection were used in this trial. First, specially designed computer templates linked to routine antenatal care 
templates were installed on all participating practices' computer systems. These facilitated anonymised data collection without the need to click on a second icon or open a separate programme to record trial data. Second, packs to facilitate data collection and recruitment were distributed to each clinician at the training sessions. These included a pack for every pregnant woman, containing: an information leaflet in English about sickle cell and thalassaemia; an information leaflet about the trial in 12 languages; a manual datacollection form for every confirmed pregnancy, to be faxed to the research team; and a summary of the research protocol. These methods were developed following work in the two pilot sites that identified the need for robust, flexible, and simple data-collection systems.

Approximately 6 months after training (which marked the end of the intervention phase of the SHIFT trial), informal face-to-face interviews were conducted with 20 GPs from practices randomised to groups 1 and 2. Part of the interviews explored GPs' views on why their practice participated in the trial. Interviews lasted approximately 30 minutes, and were held in a private room at the practice. All participants agreed to be tape-recorded.

\section{RESULTS}

Of the 123 practices in the two PCTs, four were ineligible because they did not agree to be randomised. Two practices indicated they did not agree to randomisation before expressing an interest in trial participation, and two practices indicated they did not agree to randomisation after expressing an interest in trial participation (Figure 1). Twenty-nine of the 119 eligible practices expressed an interest in participating in the trial and agreed to randomisation, giving a recruitment rate of $24 \%$ (29/119, 95\% confidence interval $[\mathrm{Cl}]=17$ to 33$)$. The recruitment process was not continued in the remaining 90 practices as the target number of practices had been exceeded $(n=24)$. There was no speculation as to how many additional practices would have participated in the trial if an active recruitment policy had been continued. Therefore, the recruitment rate of general practices to the trial is at least $24 \%$.

Twenty-seven practices participated in and completed the trial, two as pilot sites and 25 as trial sites (Figure 1). Thus, 93\% (95\% Cl $=77$ to 99) of practices were retained in the trial. Two practices withdrew because of work pressures, one prior to the start of the run-in data-collection phase and one after training was completed but before the intervention phase started.

Practices that completed the intervention phase

\begin{tabular}{|c|c|c|c|}
\hline & $\begin{array}{c}\text { Participating } \\
\text { practices }(n=27)\end{array}$ & $\begin{array}{l}\text { Non-participating } \\
\text { practices }(n=96)\end{array}$ & $P$-value \\
\hline Single-handed practice, frequency (\%) & $7(29)$ & $34(38)$ & 0.636 \\
\hline List-size per GP, median (IQR) & $\begin{array}{c}1971 \\
(1780 \text { to } 3201)\end{array}$ & $\begin{array}{c}2270 \\
\text { (1776 to } 2805)\end{array}$ & 0.989 \\
\hline $\begin{array}{l}\text { Townsend deprivation score, } \\
\text { median (IQR) }\end{array}$ & $\begin{array}{c}10.3 \\
\text { (9.7 to } 13.4)\end{array}$ & $\begin{array}{c}11.2 \\
\text { (9.5 to } 13.6)\end{array}$ & 0.694 \\
\hline $\begin{array}{l}\text { Resident percentage of minority } \\
\text { ethnic groups, median (IQR) }\end{array}$ & $\begin{array}{c}49 \\
\text { (34 to } 62)\end{array}$ & $\begin{array}{c}51 \\
\text { (36 to } 69)\end{array}$ & 0.795 \\
\hline
\end{tabular}

did not differ from the other practices in the two PCTs with respect to the number of GPs $(P=0.64)$, the list size per GP $(P=0.99)$, the Townsend deprivation score $(P=0.69)$, or the resident percentage of minority ethnic groups $(P=0.80)$ (Table 1).

\section{Trial recruitment and retention}

Qualitative analysis of interviews identified two major themes that seemed important in GPs' decisions to take part in the trial (Box 1): perceived importance of the research topic, and a general interest in research. In addition, one GP cited practical reasons for participation, while other GPs were not sure why they took part - one speculated that it might be for financial reasons. The two practices that withdrew from the study cited time pressures as the reason. One practice withdrew before starting the trial, citing general pressures in primary care at the time. The other practice withdrew after the training session because offering the test in primary care was perceived as too time consuming.

The research team synthesised the above findings into a list of factors that are potentially important in recruitment and retention for such a trial (Box 2).

\section{DISCUSSION}

\section{Summary of main findings}

The description in this article shows it is possible to recruit and retain representative primary care practices to a clinical trial, even in areas that are very socially deprived. Although it is not possible to estimate an overall recruitment rate, the study has demonstrated that the participating practices were representative of the population being investigated.

There were three key elements of the recruitment strategy that facilitated recruiting a representative sample of practices:

- The topic of the research was perceived as relevant and clinically important to the practices. The trial assessed methods of offering antenatal 


\section{Box 1. GP motivations for taking part in the trial.}

- Clinical importance:

'Because we see an awful lot of pregnant, I don't know how many pregnant people we do see but we have quite a young population. We are often seeing pregnant people, anything to improve care. You know we're quite a proactive practice anyway.' (HCP026)

'I think we thought it was a valuable project, basically, and we thought it was useful for the patients to have an increased awareness of sickle in pregnancy. We have a very high proportion of people from the relative ... the relevant ethnic minorities, so it seemed sensible for us to do it, and we're very aware of the burden of sickle cell disease in the community because we have a lot of black patients.' (HCP023)

'Mainly because we thought it was a good idea because our population we serve is a very ethnic mix and we see a lot of Afro-Caribbean, African, patients and Middle Eastern patients as well, so we thought it would be good for the women to find out early on what their status was.' (HCP020)

Interest in research:

'Well, we always try to do lots of different things, we like to engage in a bit of research, a bit of audit, a bit of service provision, enhanced care sort of thing. So that's one of the reasons. I mean, I've had a bit of a research background as well. We get a lot of requests from ... for research, people doing their degrees and many times we do help out.' (HCP009)

'... we are a research practice in a sense, we train registrars, we train medical students and I think if we feel that it's something that is worthwhile doing then we do and I think that's what we agreed after reading the initial letter.' (HCP018)

'We have been involved with other trials and we are the largest practice, and we felt it was probably going to be an important trial.' (HCP017)

- Practical reasons:

'Um ... we have a very mixed population and we have a phlebotomist on site, it's very easy for us to offer this to a patient and then they can quite easily get an appointment, it's not too much hassle for them. And we do see a lot of pregnant women, a lot ... I think it's just the population age that we have here - a lot of pregnant women.' (HCP030)

- Not sure:

'I can't remember, I don't have the original links.' (HCP032)

'I have no idea, I can't remember.' (HCP008)

'I don't think I was involved in the decision to get involved ... I think that was made while I was away so I don't know. Possibly financial.' (HCP10)

screening for sickle cell and thalassaemia, and was run in two geographical areas where $6 \%$ of pregnant women carry a significant haemoglobin variant. The invitation was signed by a local practising GP and endorsed by the local PCT. Research information sheets for practices specifically included information about how few women were offered timely sickle cell and thalassaemia screening in each PCT, that is, the problem was quantified for the local practice population.

- The method of inviting practices to participate in the trial was clear and concise. Published methods for providing key information in a systematic format were used, incorporating a timely follow-up system. An additional factor in the invitation method that may have led to a representative recruitment was the issuing of invitations to all practices in the two PCTs, rather than just those perceived to be interested in research or the topic of the trial. ${ }^{6}$

- An interest in research was identified by participating GPs as an important reason for joining the trial, even though not all participating GPs had previous experience of research.

The trial was able to pay for costs incurred by practices through participation, including the costs of time needed to attend for training. These costs were requested as part of the research grant. This approach is in keeping with the current NHS contract for GPs. GPs expect to be reimbursed for work that is outside of their core contractual obligations and the research team considered it essential to provide payment for 'non-core' work at a realistic rate. It is not possible to determine the relative importance of payment in recruitment and retention. Only one GP identified financial reward as a possible reason for participating in the trial. In keeping with this, an Australian study of GPs indicated that financial reward was the least important variable associated with interest in participating in research. ${ }^{21}$ Similarly, a recent Cochrane review reported that reimbursement for time spent on recruitment was unrelated to recruitment. ${ }^{22}$ The impression for the current trial is that the schedule or payment were related to trial retention, as discussed below.

\section{Retention}

In this case study, 93\% (27/29) of the recruited practices successfully completed the trial. It was considered that there were three key elements in the retention strategy that facilitated this:

- Good, clear communication links were established between the research team and the practices. This was maintained on a weekly basis between a nominated member of the research team and the practice link person, to ensure that any potential problems were identified and resolved rapidly by both the research team and the practice. However, it is not possible to identify the exact components of the communication strategy that were salient to good retention.

- The use of easy data-collection procedures reduced the burden on practice staff. Computerised data-collection procedures were linked to routine antenatal templates at all practices, thereby facilitating data provision and 
collection. Some practices preferred not to use computerised data-collection procedures. They were given blank forms that were completed by hand and faxed to the research team. This tailoring of data-collection procedures to practices seemed important in reducing the burden of the trial.

- The payment method was likely to have been important. The use of research-activity agreements allowed expenses to be paid as soon as pre-set targets were achieved. Interviews with GPs from each intervention practice were required by the trial protocol but some GPs found it difficult to schedule these interviews. Payment by pre-set targets seemed to encourage them to find the time for interviews that otherwise might not have achieved sufficient priority.

All three retention strategies were identified as important when working with the pilot sites, indicating the value of using pilot sites to test practical measures.

Recruiting and retaining general practices is an essential part of conducting clinical trials in primary care. Recruitment and retention of patients is also essential. The strategies used to recruit and retain patient participants in SHIFT are described elsewhere. ${ }^{23}$

\section{Strengths and limitations of the study}

This article describes a series of strategies to recruit and retain general practices in a primary care-based clinical trial. Although a retention rate of $93 \%$ is successful, it could be argued that a recruitment rate of $24 \%$ is less successful. This rate provided sufficient power to answer the research question, and was representative of the population as a whole; as such, it can be considered successful.

Qualitative data were collected from GPs in the intervention practices but not the standard care practices. As practices were allocated randomly, there is no reason to suppose that practices randomised to intervention groups were different from those randomised to standard care.

The case-study method is limited because it is only possible to describe the outcome of a multicomponent approach rather than the relative contributions of the different components. For example, it is not known whether financial compensation is needed, or the extent to which payment upon meeting pre-agreed targets is important in recruitment and retention in a trial. The case-study method does, however, act as a basis to formulate hypotheses about the most effective ways of recruiting and retaining general practices in primary care.

\section{Box 2. Possible factors associated with recruitment and} retention of general practices in a research trial.

Recruitment

Research topic:

- Topic is perceived by GPs as relevant and important. Invitation method:

- Key information for practices is presented in a systematic format.

- All practices in each PCT are invited to take part.

- Telephone follow-up within 2 weeks of invitation.

- Trial manager visits each practice interested in participating in trial.

Costs to support research process:

- Costs incurred by the practice through participation are met, including any time needed to attend for training.

Research:

- Interest and experience of research.

Retention:

Clear communication to facilitate adherence to research protocol:

- A contact person for the research is identified within each practice.

- Regular feedback is provided to the practice and any problems identified early and solutions generated jointly.

- Training sessions on the trial protocol are held at each practice.

Easy data-collection methods:

- Trial protocol is piloted.

- Use of computer template that is linked to antenatal templates.

- Participant packs are available for every pregnant woman.

Payment schedule:

- Research activity agreement is signed by the practice and research team, specifying that money is paid as targets are achieved.

\section{Comparison with existing literature}

The results of this case study are in line with the limited research on recruitment of practices to clinical trials. Foy et al reported that clear research and organisational strategies facilitate the recruitment of general practices. ${ }^{5}$ The literature on retention of practices in trials is more limited than that on practice recruitment. Successful recruitment of participants by GPs is associated with good trial organisation, along with simple documentation and trial procedures. ${ }^{13}$ Assuming that recruitment of participants is a reasonable proxy measure of retention of practices, then the findings reported by Prout et al are in line with the findings of this case study. ${ }^{13}$

\section{Implications for future research}

The hypotheses generated by this case study require testing in experimental studies. Some of the factors 
are more amenable to study than others; for example, it is possible to experimentally manipulate the invitation method and payment for participation or payment upon meeting pre-agreed targets. It is challenging to develop an intervention assessing the importance of the research topic, or communication between practice and research team, or datacollection methods, not least because it will be more difficult to maintain equipoise in these groups. Lack of equipoise has been shown to lead to poor retention in a trial. ${ }^{12}$

To conclude, three factors appeared important in recruiting practices: the research topic, invitation method, and interest in research. Three factors appeared important in retaining practices: good communication between practice and researchers, easy data-collection methods, and payment upon meeting pre-agreed targets. The effectiveness of these factors in facilitating recruitment and retention requires assessment in experimental studies.

\section{Funding body}

This study was funded by the UK Department of Health through its Health Technology Assessment Programme, grant number 03/02/03, 'Antenatal screening for haemoglobinopathies in primary care: a cluster randomised trial to inform a simulation model (Acronym: SHIFT Screening for Haemoglobinopathies in First Trimester)'. The opinions and conclusions expressed here are those of the authors and do not necessarily reflect those of the UK NHS or the Department of Health

\section{Ethical approval}

Ethical approval was granted to collect anonymised data on all pregnancies reported in participating practices (05/Q0501/36)

\section{Competing interests}

The authors have stated that there are none

\section{Acknowledgements}

We are very grateful to the general practices who participated in this study. The paper was written on behalf of the SHIFT research team: AE Ades, MRC Health Service Research Collaboration; Elizabeth N Anionwu, Thames Valley University; Karl Atkin, University of York; Katrina Brown, King's College London; Stirling Bryan, University of Birmingham; Mike Calnan, MRC Health Service Research Collaboration; Verna Davis, Manchester Sickle Cell \& Thalassaemia Centre; Moira Dick, Lambeth PCT; Martin Gulliford, King's College London; Tracey Johnston, Birmingham Women's Hospital; Patricia Jones, University College Hospital, London; Jon Karnon, University of Sheffield; Erin P Reid, King's College London; Tracy Roberts, University of Birmingham; Barbara Wild, University College Hospital, London.

\section{Discuss this article}

Contribute and read comments about this article on the Discussion Forum: http://www.rcgp.org.uk/bjgp-discuss

\section{REFERENCES}

1. Lakhani M, Baker M, Field S. The future direction of general practice: $a$ roadmap. London: Royal College of General Practitioners, 2007.

2. Yallop JJ, McAvoy BR, Croucher JL, et al. Primary health care research — essential but disadvantaged. Med J Aust 2006; 185(2): 118-120.

3. Huibers MJH, Bleijenberg G, Beurskens AJHM, et al. An alternative trial design to overcome validity and recruitment problems in primary care research. Fam Pract 2004; 21(2): 213-218.

4. Wilson S, Delaney BC, Roalfe A, et al. Randomised controlled trials in primary care: case study. BMJ 2000; 321(7252): 24-27.

5. Foy R, Parry J, Duggan A, et al. How evidence based are recruitment strategies to randomized controlled trials in primary care? Experience from seven studies. Fam Pract 2003; 20(1): 83-92.

6. Ellis SD, Bertoni AG, Bonds DE, et al. Value of recruitment strategies used in a primary care practice based trial. Contemp Clin Trials 2007; 28(3): 258-267.

7. Bateman H. A Research Information Sheet of Practices (RISP): a too to facilitate research participation. Fam Pract 2002; 19(6): 691-697.

8. Hoddinott P, Britten J, Harrild K, Godden DJ. Recruitment issues when primary care population clusters are used in randomised controlled trials: climbing mountains or pushing boulders uphill? Contemp Clin Trials 2007; 28(3): 232-241.

9. Shelton BJ, Wofford JL, Gosselink CA, et al. Recruitment and retention of physicians for primary care research. J Commun Health 2002; 27(2): 79-89.

10. Fransen GAJ, van Marrewijk CJ, Mujakovic S, et al. Pragmamtic trials in primary care. Methodological challenges and solutions demonstrated by the DIAMOND-study. BMC Med Res Methodol 2007; 7: 16.

11. Farrin A, Russell I, Torgerson D, Underwood M. Differential recruitment in a cluster randomized trial in primary care: the expererince of the UK Back pain, Exercise, Active management and Manipulation (UK BEAM) feasibility study. Clin Trials 2005; 2(2): 119-124.

12. Hetherton J, Matheson A, Robson M. Recruitment by GPs during consultations in a primary care randomized controlled trial comparing computerized psychological therapy with clinical psychology and routine care: problems and possible solutions. Prim Health Care Res Dev 2004; 5(1): 5-10.

13. Prout H, Butler C, Kinnersley P, et al. A qualitative evaluation of implementing a randomized controlled trial in general practice. Fam Pract 2003; 20(6): 675-681.

14. Medical Research Council. Medical Research Council's General Practice Research Framework. London: MRC, 2007. http://www.gprf.mrc.ac.uk (accessed 18 Mar 2008).

15. UK Clinical Research Network. Primary Care Research Network. http://www.ukcrn.org.uk/index/networks/primarycare (accessed 18 Mar 2008).

16. Marteau TM. Protocol 06PRT/921: Antenatal screening for haemoglobinopathies in primary care: a cluster randomised trial to inform a simulation model. SHIFT (Screening for Haemoglobinopathies In First Trimester) Trial. London: Lancet, 2008. http://www.thelancet.com/journals/lancet/misc/protocol/06PRT-921 (accessed 4 Jul 2008).

17. Dormandy E, Gulliford M, Reid EP, et al. Delay between pregnancy confirmation and sickle cell thalassaemia screening: a populationbased cohort study. Br J Gen Pract 2008; 58(548): 154-159.

18. Office for National Statistics. National Statistics. Neighbourhood Statistics. http://www.neighbourhood.statistics.gov.uk/dissemination/ LeadTableView.do;jsessionid=ac1 f930bce68ce4b9484e3d40548e7dfdc 304ealb46.e38PbNqOa3qRe3eOahqLaxuLe6fznA5Pp7ftolbGmkTy?a $=7 \& \mathrm{~b}=276767 \& \mathrm{c}=$ newham $\& \mathrm{~d}=13 \& \mathrm{e}=10 \& \mathrm{~g}=343478 \& \mathrm{i}=1001 \mathrm{x} 1003 \mathrm{x}$ $1004 \& \mathrm{o}=35 \& \mathrm{~m}=0 \& \mathrm{r}=1 \& \mathrm{~s}=1201083025343 \&$ enc $=1 \& \mathrm{dsFamilyId}=72$ $4 \&$ bhcp $=1$ (accessed 30 Apr 2008).

19. NHS Sickle Cell and Thalassaemia Programme. NHS Sickle Cell and Thalassaemia Programme: information for community practitioners. London: NHS Sickle Cell and Thalassaemia Programme, 2005. http://sct.screening.nhs.uk/Documents/CommPracInfo.pdf (accessed 18 Mar 2008).

20. NHS Sickle Cell and Thalassaemia Screening Programme. Standards for linked antenatal and newborn screening programme. London: UK National Screening Committee, 2006

21. Silagy CA, Carson NE. Factors affecting the level of interest and activity in primary care research among general practitioners. Fam Pract 1989; 6(3): 173-176.

22. Rendell JM, Merritt RK, Geddes JR. Incentives and disincentives to participation by clinicians in randomised controlled trials (review). Cochrane Database Syst Rev 2007; 2: 1-19.

23. Dormandy E, Brown K, Reid EP, Marteau TM. Towards socially inclusive research: an evaluation of telephone questionnaire administration in a multilingual population. BMC Med Res Methodol $2008 ; 8(1): 2$ 


\section{Appendix 1. SHIFT (screening for haemoglobinopathies in first trimester) trial.}

Purpose:

To examine the feasibility, acceptability, and effectiveness of offering antenatal screening for sickle cell and thalassaemia in one of three ways:

Group 1: In primary care, when women first report their pregnancies with partners offered testing at the same time;

Group 2: In primary care, when women first report their pregnancies with partners offered testing later and only if women are identified as carriers;

Group 3: In community-based secondary care, when women are booked by midwives with partners offered testing later and only if women are identified as carriers.

Practices will be randomised to offer screening in one way only.

To model the cost-effectiveness of these three methods of offering screening.

Context: Many women are not making informed choices about antenatal screening for sickle cell and thalassaemia because they are not offered the test or they are offered the test too late in pregnancy. For example, in a recent audit in Newham in 2002, 59 couples were at risk of having an affected baby, but only nine had been screened by 11 weeks gestation.

Practice Involvement (Details in Supplementary Information 2).

The practice will:

- offer antenatal screening for sickle cell and thalassaemia to all pregnant women when the pregnancy is first confirmed, and;

provide anonymous data on the number of pregnancies and time of offer and uptake of antenatal screening for sickle cell and thalassaemia.

Ethical Committee Approval: Ethical and R\&D approval for the Trial has been obtained.

Period of data collection: 6 months, plus retrospective data on number of pregnancies and time of screening for 6 months prior to data collection period.

Suggested start date in this practice: March 2006.

Practice costs are reimbursed: Yes, approximately $£ 3000$. This covers administrative costs of providing anonymised data, costs of offering the screening test to pregnant women, and locum costs for attending a training session.

Your project contacts:

Name: Elizabeth Dormandy, SHIFT Trial Manager

Phone: 02071880192

Email: elizabeth.dormandy@kcl.ac.uk

Lead researcher: Professor Theresa Marteau, Professor of Health Psychology

Host institution: King's College London

Funder: NHS R\&D Health Technology Assessment Programme

What the researcher will do:

- Provide nationally approved training in offering antenatal screening for sickle cell and thalassaemia and information for pregnant women and their partners about the screening process;

Seek consent from women to take part in the evaluation of the trial;

Conduct trial evaluations;

Maintain contact with local midwives, counsellors, laboratory staff and obstetricians.

To undertake the research the researcher will request access to the following personnel, records and/or practice facilties:

- Anonymised data on the number of pregnancies and the offer and time of screening for all pregnant women for a 12-month period (6 months prior to data collection and during the data collection period);

Contact details of pregnant women who agree to be contacted by a researcher.

What the practice personnel will be asked to do:

Agree to randomisation to one of three patterns of care;

continued ... 


\section{Appendix 1 continued. SHIFT (screening for haemoglobinopathies in first trimester) trial.}

Every GP to attend a training session in (i) offering antenatal screening for sickle cell and thalassaemia (ii) the trial protocol (approximately 2 hours, provided at the practice);

- Provide data on the number of pregnancies and the offer and time of screening uptake for all pregnant women for a 12 month period;

- Offer screening to all eligible women for 6 months of the data collection period. Eligible women are those confirming pregnancy, aged 18 years and over, wanting to proceed with the pregnancy and whose carrier status is not documented in primary care records. Practices randomised to Group 1 to offer screening to partners of eligible women;

- Seek permission for a researcher to contact pregnant women. One GP from each practice to participate in an interview about the experiences of offering screening in primary care;

Local sickle cell and thalassaemia counsellors will provide the support pathway for any carriers that are identified through SHIFT.

How consent and confidentiality will be handled:

- The proposed research will be conducted in accord with the Research Governance Framework, COREC, LREC and PCT approvals;

- A researcher will only approach women who agree to be contacted. Women will be informed about the risks and benefits of study participation before consent to participate in the evaluation is sought.

Practice feedback:

- This will be provided by personal contacts with Elizabeth Dormandy and trial updates via SHIFT website. Feedback of results prior to presentation to the scientific community will be offered to practices either as a seminar held at the practice or a written report in the summer of 2007.

We thank you for your help and interest.

Please contact Elizabeth Dormandy if you would like any further information or clarification (email elizabeth.dormandy@kcl.ac.uk or tel 0207188 0192)

This Research Information Sheet for Practices was initiated by the General Practice \& Primary Care Research Unit, Cambridge University. Version 1.3: October 2005 